\title{
Laplacians and the Cheeger inequality for directed graphs *
}

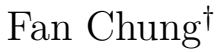 \\ University of California, San Diego \\ La Jolla, CA 92093-0112
}

\begin{abstract}
We consider Laplacians for directed graphs and examine their eigenvalues. We introduce a notion of a circulation in a directed graph and its connection with the Rayleigh quotient. We then define a Cheeger constant and establish the Cheeger inequality for directed graphs. These relations can be used to deal with various problems that often arise in the study of non-reversible Markov chains including bounding the rate of convergence and deriving comparison theorems.
\end{abstract}

\section{Introduction}

There is a large literature on the eigenvalues of the Laplacians for undirected graphs $[1,2,6]$. In contrast, the spectral approach for directed graphs has not been as well developed. For example, one of the key invariants for undirected graphs is the Cheeger constant, which is sometimes called conductance. The relation between the Cheeger constant and eigenvalues of the Laplacian of an (undirected) graph is represented by the Cheeger inequality, which is one of the main tools for bounding the mixing time for random walks on undirected graphs. Many applications in communication networks and in approximation algorithms involve directed graphs. The goal of this paper is to introduce the Laplacian of a directed graph and establish a Cheeger inequality for a directed graph.

Motivated by non-reversible Markov chains, Fill [10] derived bounds for the rate of convergence by using eigenvalues of certain Hermitian matrices associated with a directed graph, such as the sum and product of the transition probability

\footnotetext{
* Annals of Combinatorics, to appear.

${ }^{\dagger}$ Research supported in part by NSF Grants DMS 0100472 and ITR 0205061
} 
matrix and its transpose. The Cheeger inequality for directed graphs provides methods for further bounding the rate of convergence.

We will define the Laplacian of a graph as a Hermitian matrix by using the transition probability matrix. As we will see in Section 4, the spectral gap of the Laplacian is related to the real parts of the eigenvalues of the transition probability matrix. This connection will be illustrated through a natural relation between the Laplacian and the Rayleigh quotient. One essential notion in directed graphs that differs from the undirected case, is the inference of a circulation of a directed graph (defined in Section 3). By using circulations, we can define a Cheeger constant and derive a Cheeger inequality for a directed graph. Consequently, we can use the Cheeger constant to bound eigenvalues of the Laplacian and vice versa. In Section 7, we will use eigenvalues of the Laplacian to bound the rate of convergence of lazy random walk as a non-reversible Markov chain. Thus, the Cheeger constant can then be used to bound the rate of convergence for lazy random walks for directed graphs as well. In Section 8, several versions of comparison theorems are given.

\section{Preliminaries}

Suppose $G$ is a directed graph $G$ with vertex set $V(G)$ and edge set $E(G)$. For a directed edge $(u, v)$ in $E(G)$, we say that there is an edge $(u, v)$ from $u$ to $v$, or, $u$ has an out-neighbor $v$, or, $v$ has an in-neighbor $u$. The number of out-neighbors of $u$ is the out-degree of $u$, denoted by $d_{u}^{\text {out }}$ or $d_{u}$. Similarly, the number of in-neighbors of $v$ is denoted by $d_{v}^{i n}$. A walk is a sequence of vertices $w=\left(v_{0}, v_{1}, \ldots, v_{s}\right)$ such that $\left(v_{i-1}, v_{i}\right)$ is an edge.

A random walk is defined by a transition probability matrix $P$, where $P(u, v)$ denotes the probability of moving from vertex $u$ to vertex $v$. Clearly, $P(u, v)>0$ only if $(u, v)$ is an edge. Also, $\sum_{v} P(u, v)=1$. However, for a directed graph, it is not required that $\sum_{u} P(u, v)=1$ in general. This is different from the undirected case.

For a given directed graph $G$, a typical transition probability matrix $P=P_{G}$ is

$$
P(u, v)= \begin{cases}\frac{1}{d_{u}} & \text { if }(u, v) \text { is an edge } \\ 0 & \text { otherwise. }\end{cases}
$$

Unless stated otherwise, we will assume that $P$ is of this form.

For a weighted directed graph with edge weights $w_{u v} \geq 0$, a general transition probability matrix $P$ can be defined as

$$
P(u, v)=\frac{w_{u v}}{\sum_{z} w_{u z}}
$$


An unweighted directed graph is just a special case with weight having value 1 or 0 .

The Perron-Frobenius Theorem [9] states that an irreducible matrix $M$ with non-negative entries has a unique (left) eigenvector with all entries positive. Let $\rho$ denote the eigenvalue of the all positive eigenvector of $P$. Then, the absolute values of all eigenvalues of $M$ are bounded above by $\rho$.

This can be translated to language for directed graphs. Namely, the transition probability matrix $P$ of a strongly connected directed graph has a unique left eigenvector $\phi$ with $\phi(v)>0$ for all $v$, and

$$
\phi P=\rho \phi .
$$

Here we treat $\phi$ as a row vector. Since

$$
P 1=1,
$$

we have $\rho=1$ and the Perron-Frobenius Theorem implies that all other eigenvalues of $P$ have absolute value at most 1 . We can normalize and choose $\phi$ satisfying

$$
\sum_{v} \phi(v)=1
$$

We will call $\phi$ the Perron vector of $P$. For a general directed graph, there is no closed form solution for $\phi$ (see [5]). Nevertheless, there is a polynomial-time algorithm to evaluate $\phi$ computationally.

If there is more than one eigenvalue of $P$ with absolute value 1 , it is not too difficult to show that the random walk is periodic and the g.c.d. of the lengths of all directed cycles in the graph is greater than 1 . If $G$ is strongly connected and aperiodic, the random walk converges to the stationary distribution $\phi$, the Perron vector.

Example 1: An undirected graph can be viewed as a directed graph with directed edges $(u, v),(v, u)$ for each undirected edge $\{u, v\}$. It is easy to show that $\phi(v)=d_{v} / \sum_{u} d_{u}($ see $[6])$.

Example 2: For a directed regular graph on $n$ vertices with in-degrees and out-degrees all equal, we have $\phi=1 / n$.

Example 3: A directed graph is called Eulerian if the in-degree of each vertex is equal to its out-degree. For an Eulerian graph, its Perron vector $\phi$ is proportional to the out-degree sequence, i.e., $\phi(v)=d_{v} / \sum_{u} d_{u}=d_{v} / m$ where $m$ is the total number of edges.

For a strongly connected graph $G$ on $n$ vertices, the Perron vector $\phi$ can have coordinates with values exponentially small, as indicated in the following example. 
Example 4: We consider a directed graph on $n$ vertices formed by the union of a directed cycle $C_{n}$ consisting of edges $\left(v_{j}, v_{j+1}\right)$ (where the indices are taken modulo $n)$ and $n-1$ edges $\left(v_{j}, v_{1}\right)$, for $j=1, \ldots, n-1$. The Perron vector $\phi$ has an exponentially decreasing distribution. Namely, $\phi\left(v_{j}\right)=2^{-j+1} \phi\left(v_{1}\right)$ for $j \geq 1$ and $\phi\left(v_{1}\right)=1 /\left(2-2^{-n+1}\right)$.

For a strongly connected graph $G$ on $n$ vertices, the Perron vector $\phi$ of $G$ satisfies

$$
\max _{x} \phi(x) \leq k^{D} \min _{y} \phi(y)
$$

where $D$ denotes the diameter of $G$ and $k$ denotes the maximum out-degree.

\section{The circulation of a directed graph}

In a directed graph $G$, we consider a function $F: E(G) \rightarrow \mathbb{R}^{+} \cup\{0\}$ that assigns to each directed edge $(u, v)$ a non-negative value $F(u, v) . F$ is said to be a circulation if at each vertex $v$, we have

$$
\sum_{\substack{u \\ u \rightarrow v}} F(u, v)=\sum_{\substack{w \\ v \rightarrow w}} F(v, w) .
$$

For example, if $G$ contains a directed cycle $C_{n}$. A function that assigns 1 to each of the edges in $C_{n}$ and 0 otherwise is a circulation.

We remark that the term of circulation has been previously used in network flow theory, usually with specified sources and sinks. Here we define the circulation in a directed graph in a natural way without distinct sources or sinks.

Lemma 1 For a directed graph $G$, the eigenvector $\phi$ of the transition probability matrix $P$ having eigenvalue 1 is associated with a circulation $F_{\phi}$ as follows: For $(u, v) \in E(G)$,

$$
F_{\phi}(u, v)=\phi(u) P(u, v) .
$$

Proof: To see that $F_{\phi}$ is a circulation, it can be easily checked that

$$
\begin{aligned}
\sum_{\substack{u \rightarrow v \\
u \rightarrow v}} F_{\phi}(u, v) & =\sum_{\substack{u \rightarrow v \\
u \rightarrow v}} \phi(u) P(u, v) \\
& =\phi(v) \\
& =\sum_{\substack{w \\
v \rightarrow w}} \phi(v) P(v, w) \\
& =\sum_{\substack{w \\
v \rightarrow w}} F_{\phi}(v, w) .
\end{aligned}
$$


A circulation is said to be reversible if

$$
F(u, v)=F(v, u) .
$$

Clearly, a reversible random walk satisfies (1) and has a reversible circulation. An undirected graph has a reversible circulation and thus the random walk on an undirected graph is a reversible Markov chain.

For a circulation $F_{\phi}$, the flow at a vertex $v$ is given by

$$
\phi(v)=\sum_{\substack{u \\ u \rightarrow v}} F(u, v)=\sum_{\substack{w \\ v \rightarrow w}} F(v, w) .
$$

For some directed graphs, the flows at the vertices can differ by exponential factors as seen in the graphs of Example 4. For undirected graphs, the flow at a vertex $v$ is proportional to its degree and is within a polynomial factor (of $n$ ) of the flow at any other vertex. As we will see, this fact has significant consequences in studying random walks and isoperimetric inequalities for directed graphs.

\section{The Rayleigh quotient and the Laplacian for a directed graph}

In this section, we give the definitions of the Laplacian and the Rayleigh quotient for a directed graph. For two vectors $f$ and $g$, we denote the usual inner product $\langle f, g\rangle=\sum_{v} f(v) \bar{g}(v)$.

For a directed graph $G$ with transition probability matrix $P$ and the Perron vector $\phi$, the Rayleigh quotient for any $f: V(G) \rightarrow \mathbb{C}$, is defined as follows.

$$
R(f)=\frac{\sum_{u \rightarrow v}|f(u)-f(v)|^{2} \phi(u) P(u, v)}{\sum_{v}|f(v)|^{2} \phi(v)}
$$

The Laplacian of a directed graph $G$ is defined by

$$
\mathcal{L}=I-\frac{\Phi^{1 / 2} P \Phi^{-1 / 2}+\Phi^{-1 / 2} P^{*} \Phi^{1 / 2}}{2}
$$

where $\Phi$ is a diagonal matrix with entries $\Phi(v, v)=\phi(v)$ and $M^{*}$ denotes the conjugated transpose of $M$. Clearly, the Laplacian satisfies

$$
\mathcal{L}^{*}=\mathcal{L} .
$$

We also define the combinatorial Laplacian $L$.

$$
L=\Phi-\frac{\Phi P+P^{*} \Phi}{2} .
$$


We wish to show the following:

Theorem 1 For a directed graph $G$ with the transition probability matrix $P$, suppose the Rayleigh quotient and the Laplacian are defined as above. Then we have

$$
\begin{aligned}
R(f) & =2 \frac{\langle f L, f\rangle}{\langle f \Phi, f\rangle} \\
& =2-\frac{f\left(\Phi P+P^{*} \Phi\right) f^{*}}{f \Phi f^{*}} \\
& =2 \frac{\langle g \mathcal{L}, g\rangle}{\|g\|^{2}}
\end{aligned}
$$

where $g=f \Phi^{1 / 2}$.

Proof: We can write

$$
\begin{aligned}
R(f) & =\frac{\sum_{u \rightarrow v}|f(u)-f(v)|^{2} \phi(u) P(u, v)}{\sum_{v}|f(v)|^{2} \phi(v)} \\
& =\frac{\sum_{u \rightarrow v}(f(u)-f(v))(\bar{f}(u)-\bar{f}(v)) \phi(u) P(u, v)}{f \Phi f^{*}} \\
& =\frac{2 f \Phi f^{*}-\sum_{u \rightarrow v}(f(u) \phi(u) P(u, v) \bar{f}(v)+\bar{f}(u) \phi(u) P(u, v) f(v))}{f \Phi f^{*}} \\
& =2-\frac{f\left(\Phi P+P^{*} \Phi\right) f^{*}}{f \Phi f^{*}} \\
& =2 \frac{f L f^{*}}{f \Phi f^{*}} \\
& =2 \frac{\langle f L, f\rangle}{\langle f \Phi, f\rangle} .
\end{aligned}
$$

Furthermore, by taking $g=f \Phi^{1 / 2}$, we have

$$
\begin{aligned}
R(f) & =2-\frac{f\left(\Phi P+P^{*} \Phi\right) f^{*}}{f \Phi f^{*}} \\
& =2-\frac{g\left(\Phi^{1 / 2} P \Phi^{-1 / 2}+\Phi^{-1 / 2} P^{*} \Phi^{1 / 2}\right) g^{*}}{g g^{*}} \\
& =2 \frac{g \mathcal{L} g^{*}}{g g^{*}} \\
& =2 \frac{\langle g \mathcal{L}, g\rangle}{\|g\|^{2}}
\end{aligned}
$$

as desired.

As an immediate consequence, we have the following: 
Corollary 1 Suppose a directed graph $G$ has Laplacian eigenvalues $0=\lambda_{0} \leq$ $\lambda_{1} \leq \ldots \leq \lambda_{n-1}$. Then the eigenvalues and the Rayleigh quotient are related as follows:

1. For $\lambda_{1}$, we have

$$
\begin{aligned}
\lambda_{1} & =\inf _{\substack{f \\
\sum_{x} f(x) \phi(x)=0}} \frac{R(f)}{2} \\
& =\inf _{f} \sup _{c} \frac{\sum_{u \rightarrow v}|f(u)-f(v)|^{2} \phi(u) P(u, v)}{2 \sum_{v}|f(v)-c|^{2} \phi(v)}
\end{aligned}
$$

where $\phi$ is the Perron vector and $\Phi$ is the Perron diagonal matrix.

2. Suppose $\phi_{i}$ is an eigenvector of the Laplacian associated with eigenvalue $\lambda_{i}$. For $f_{i}=\phi_{i} \Phi^{-1 / 2}$, we have

$$
\begin{aligned}
\lambda_{i} f_{i}(x) \phi(x) & =\left[f_{i}\left(\Phi-\frac{\Phi P+P^{*} \Phi}{2}\right)\right](x) \\
& =\phi(x) f_{i}(x)-\frac{\sum_{y \rightarrow x} f_{i}(y) \phi(y) P(y, x)}{2}-\frac{\sum_{x \rightarrow y} f_{i}(y) P(x, y) \phi(x)}{2} \\
& =\frac{1}{2} \sum_{y}\left(f_{i}(x)-f_{i}(y)\right)(\phi(y) P(y, x)+P(x, y) \phi(x))
\end{aligned}
$$

for each vertex $x$.

Theorem 2 For a directed graph $G$, the eigenvalue $\lambda_{1}$ of the Laplacian $\mathcal{L}$ is related to the eigenvalues $\rho_{i}$ of the transition probability matrix $P$ as follows:

$$
\lambda_{1} \leq \min _{i \neq 0}\left(1-R e \rho_{i}\right)
$$

where Re $x$ denotes the real part of the complex number $x$.

Proof: Suppose $f \Phi$ is an eigenvector of $P$ with eigenvalue $\rho_{i}$. Clearly $\bar{f} \Phi$ is an eigenvector of $P$ with eigenvalue $\bar{\rho}_{i}$. From Theorem 1, we have

$$
\begin{aligned}
R(f) & =2-\frac{f\left(\Phi P+P^{*} \Phi\right) f^{*}}{f \Phi f^{*}} \\
& =2-\frac{f \Phi P f^{*}+\bar{f} \Phi P \bar{f}^{*}}{f \Phi f^{*}} \\
& =2-\frac{\rho_{i} f \Phi f^{*}+\bar{\rho}_{i} f \Phi f^{*}}{f \Phi f^{*}} \\
& =2\left(1-\operatorname{Re} \rho_{i}\right) .
\end{aligned}
$$

On the other hand, we have

$$
R(f)=2 \frac{\langle g \mathcal{L}, g\rangle}{\|g\|^{2}}
$$


for $g=f \Phi^{1 / 2}$. Hence,

$$
\begin{aligned}
\lambda_{1} & =\inf _{\substack{g \\
\left\langle g, \mathbf{1} \Phi^{1 / 2}\right\rangle=0}} \frac{\langle g \mathcal{L}, g\rangle}{\|g\|^{2}} \\
& =\inf _{\substack{f \\
\langle f \Phi, \mathbf{1}\rangle=0}} \frac{R(f)}{2} \\
& \leq \min _{i \neq 0}\left(1-\operatorname{Re} \rho_{i}\right) .
\end{aligned}
$$

\section{The Cheeger constant and the Cheeger in- equality for a directed graph}

In a directed graph $G$, let $S$ denote a subset of vertices. The out-boundary of $S$, denoted by $\partial S$, consists of all edges $(u, v)$ with $u \in S$ and $v \notin S$. We write

$$
F(\partial S)=\sum_{u \in S, v \notin S} F(u, v) .
$$

If $F$ is a circulation, it follows from the definition that

$$
F(\partial S)=F(\partial \bar{S})
$$

where $\bar{S}$ denotes the complement of $S$.

For a vertex $v$, we define

$$
F(v)=\sum_{u, u \rightarrow v} F(u, v)
$$

and

$$
F(S)=\sum_{v \in S} F(v) .
$$

For a strongly connected graph $G$ with stationary distribution $\phi$, we consider the circulation flow $F_{\phi}$ and define the Cheeger constant as follows:

$$
h(G)=\inf _{S} \frac{F_{\phi}(\partial S)}{\min \left\{F_{\phi}(S), F_{\phi}(\bar{S})\right\}} .
$$

where $S$ ranges over all non-empty proper subset of the vertex set of $G$. We can relate $h$ to the eigenvalues of the Laplacian by establishing the directed analog of the Cheeger inequality. 
Theorem 3 For a directed graph $G$ with eigenvalues $\lambda_{i}$ of the Laplacian. Then $\lambda=\min _{i \neq 0}\left|\lambda_{i}\right|$ satisfies

$$
2 h(G) \geq \lambda \geq \frac{h^{2}(G)}{2}
$$

where $h(G)$ is the Cheeger constant of $G$.

Proof: $\quad$ To see that $h=h(G) \geq \lambda / 2$, we consider the set $S$ which achieves the Cheeger constant in (2), i.e.,

$$
h=\frac{F_{\phi}(\partial S)}{F_{\phi}(S)} .
$$

We define $f: V(G) \rightarrow \mathbb{C}$ as follows:

$$
f(v)= \begin{cases}\frac{1}{F_{\phi}(S)} & \text { if } v \in S \\ -\frac{1}{1-F_{\phi}(S)} & \text { otherwise. }\end{cases}
$$

Clearly, $\phi \cdot f=0$. From Theorem 1, we have

$$
\begin{aligned}
\lambda & =\inf _{\substack{\varphi \\
\phi \cdot \varphi=0}} \frac{1}{2} R(\varphi) \\
& \leq \frac{1}{2} \frac{\sum_{u \rightarrow v}(f(u)-f(v))^{2} \phi(u) P(u, v)}{\sum_{v} f^{2}(v) \phi(v)} \\
& =\frac{\left(\frac{1}{F_{\phi}(S)}+\frac{1}{1-F_{\phi}(S)}\right)^{2} F_{\phi}(\partial S)}{\frac{1}{F_{\phi}(S)}+\frac{1}{1-F_{\phi}(S)}} \\
& =\frac{F_{\phi}(\partial S)}{F_{\phi}(S)\left(1-F_{\phi}(S)\right)} \\
& \leq \frac{2 F_{\phi}(\partial S)}{F_{\phi}(S)} \\
& =2 h
\end{aligned}
$$

To prove $\lambda \geq h^{2} / 2$, we consider the eigenvector $\phi_{1}$ of the Laplacian $\mathcal{L}$ which achieves $\lambda$. Since $\mathcal{L}$ is symmetric, we may assume that $\phi$ is real-valued. For $f(x)=\phi_{1}(x) \phi(x)^{-1 / 2}$, from Corollary 1 , we have

$$
\lambda f(x) \phi(x)=\frac{1}{2} \sum_{y}(f(x)-f(y))(\phi(y) P(y, x)+P(x, y) \phi(x))
$$

Without loss of generality, we may assume

$$
f\left(v_{1}\right) \geq f\left(v_{2}\right) \geq \ldots \geq f\left(v_{n}\right)
$$

and

$$
\sum_{f(v)<0} \phi(v) \geq \sum_{f(u) \geq 0} \phi(u) .
$$


From the definition of the Cheeger constant, for each fixed $i$ with $f\left(v_{i}\right) \geq 0$, we have

$$
h \sum_{j \leq i} \phi\left(v_{j}\right) \leq \sum_{k \leq i<l} \phi\left(v_{k}\right) P\left(v_{k}, v_{l}\right) .
$$

We denote

$$
V_{+}=\{v: f(v) \geq 0\}
$$

and define

$$
g(x)= \begin{cases}f(x) & \text { if } x \in V_{+} \\ 0 & \text { otherwise. }\end{cases}
$$

Then we have

$$
\begin{aligned}
& \lambda=\frac{\sum_{x \in V_{+}} f(x) \sum_{y}(f(x)-f(y))(\phi(y) P(y, x)+P(x, y) \phi(x))}{2 \sum_{x \in V_{+}} f(x)^{2} \phi(x)} \\
& \geq \frac{\sum_{x} g(x) \sum_{y}(g(x)-g(y))(\phi(y) P(y, x)+P(x, y) \phi(x))}{2 \sum_{x} g(x)^{2} \phi(x)} \\
& =\frac{\sum_{x} \sum_{y}(g(x)-g(y))^{2} \phi(y) P(y, x)}{2 \sum_{x} g(x)^{2} \phi(x)} \\
& =\frac{\left(\sum_{x} \sum_{y}(g(x)-g(y))^{2} \phi(y) P(y, x)\right)\left(\sum_{x} \sum_{y}(g(x)+g(y))^{2} \phi(y) P(y, x)\right)}{2 \sum_{x} g(x)^{2} \phi(x)\left(\sum_{x} \sum_{y}(g(x)+g(y))^{2} \phi(y) P(y, x)\right)} \\
& \geq \frac{\left(\sum_{x} \sum_{y}\left|g(x)^{2}-g(y)^{2}\right| \phi(y) P(y, x)\right)^{2}}{8\left(\sum_{x} g(x)^{2} \phi(x)\right)^{2}} \\
& =\frac{\left(\sum_{k} \sum_{l>k}\left(g\left(v_{k}\right)^{2}-g\left(v_{l}\right)^{2}\right)\left(\phi\left(v_{l}\right) P\left(v_{l}, v_{k}\right)+\phi\left(v_{k}\right) P\left(v_{k}, v_{l}\right)\right)^{2}\right.}{8\left(\sum_{x} g(x)^{2} \phi(x)\right)^{2}} \\
& =\frac{\left(\sum_{k}\left(g\left(v_{k}\right)^{2}-g\left(v_{k+1}\right)^{2}\right) \sum_{i \leq k<j}\left(\phi\left(v_{i}\right) P\left(v_{i}, v_{j}\right)+\phi\left(v_{j}\right) P\left(v_{j}, v_{i}\right)\right)^{2}\right.}{8\left(\sum_{x} g(x)^{2} \phi(x)\right)^{2}} \\
& \geq \frac{\left(\sum_{k}\left(g\left(v_{k}\right)^{2}-g\left(v_{k+1}\right)^{2}\right) 2 h \sum_{i \leq k} \phi\left(v_{i}\right)\right)^{2}}{8\left(\sum_{x} g(x)^{2} \phi(x)\right)^{2}} \\
& =\frac{h^{2}\left(\sum_{i} \phi\left(v_{i}\right) \sum_{k \geq i}\left(g\left(v_{k}\right)^{2}-g\left(v_{k+1}\right)^{2}\right)\right)^{2}}{2\left(\sum_{x} g(x)^{2} \phi(x)\right)^{2}} \\
& =\frac{h^{2}\left(\sum_{i} \phi\left(v_{i}\right) g\left(v_{i}\right)^{2}\right)^{2}}{2\left(\sum_{x} g(x)^{2} \phi(x)\right)^{2}} \\
& =\frac{h^{2}}{2} \text {. }
\end{aligned}
$$




\section{Bounds for Cheeger constants}

The following lower bounds for the Cheeger constants for various families of directed graphs follow from the upper bounds for $\phi$.

Lemma 2 For a strongly connected regular directed graph $G$ on $n$ vertices and degree $k$, we have

$$
h(G) \geq \frac{2}{k n} .
$$

Proof: We have

$$
\begin{aligned}
h(G) & =\inf _{S} \frac{F_{\phi}(\partial S)}{\min \left\{F_{\phi}(S), F_{\phi}(\bar{S})\right\}} \\
& =\inf _{S} \frac{|\partial S|}{k n \min \left\{F_{\phi}(S), F_{\phi}(\bar{S})\right\}} \\
& =\inf _{S} \frac{|\partial S|}{k \min \{|S|,|\bar{S}|\}} \\
& \geq \frac{2}{k n} .
\end{aligned}
$$

Lemma 3 For a strongly connected Eulerian directed graph $G$ on $m$ edges, we have

$$
h(G) \geq \frac{2}{m} .
$$

Proof: As before, we have

$$
\begin{aligned}
h(G) & =\inf _{S} \frac{F_{\phi}(\partial S)}{\min \left\{F_{\phi}(S), F_{\phi}(\bar{S})\right\}} \\
& \geq \inf _{S} \frac{1 / m}{\min \left\{F_{\phi}(S), F_{\phi}(\bar{S})\right\}} \\
& \geq \frac{2}{m} .
\end{aligned}
$$

Lemma 4 For some directed graphs $G$ with bounded out-degrees, the Cheeger constant of $G$ can be exponentially small, i.e.,

$$
h(G) \leq c^{-n}
$$

for some constant $c$. 
Proof: To see this, we consider a graph which is formed in a similar way as the graph in Example 4. We construct a graph $G$ with vertex set $\left\{v_{1}, \ldots, v_{2 N}\right\}$. There are two directed cycles $\left(v_{1}, \ldots, v_{N}\right)$ and $\left(v_{2 N}, \ldots, v_{N+1}\right)$. There are edges $\left(v_{j}, v_{1}\right)$, for $1 \leq j \leq N$ and $\left(v_{k}, v_{2 N}\right)$ for $N<k \leq 2 N$ as well as edges $\left(v_{N}, v_{N+1}\right)$ and $\left(v_{N+1}, v_{N}\right)$. We can see that for $i=2, \ldots, N-1$,

$$
\phi\left(v_{i}\right)=\phi\left(v_{2 N-i+1}\right)=\frac{1}{2} \phi\left(v_{i-1}\right) .
$$

Thus, using $S=\{1, \ldots, N\}$, we have

$$
\begin{aligned}
h(G) & \leq \frac{\phi\left(v_{N}\right) / 2}{\sum_{j \leq N} \phi\left(v_{j}\right)} \\
& \leq \frac{1}{2^{N}}
\end{aligned}
$$

as desired.

From Lemma 4 and the Cheeger inequality, we have the following.

Theorem 4 Some strongly connected directed graphs have Laplacian eigenvalues that are exponenentially small (in the number of vertices of the graph).

We remark that for a connected undirected graph on $n$ vertices, the eigenvalue $\lambda_{1}$ of the Laplacian is at least $1 / n^{2}$. Hence here is a real difference between the directed and undirected graphs.

\section{The rate of convergence of random walks on directed graphs}

In the study of rapidly mixing Markov chains, the convergence in the $L_{2}$ distance is rather weak since it does not require convergence to the stationary distribution at every vertex. A strong notion of convergence that is often used is measured by the so-called total variation distance. After $s$ steps the total variation distance of $P$ to the stationary distribution $\phi(x)$ is given by

$$
\begin{aligned}
\Delta_{T V}(s) & =\max _{A \subset V(G)} \max _{y \in V(G)}\left|\sum_{x \in A}\left(P^{s}(y, x)-\phi(x)\right)\right| \\
& =\frac{1}{2} \max _{y \in V(G)} \sum_{x \in V(G)}\left|P^{s}(y, x)-\phi(x)\right| .
\end{aligned}
$$

Another notion of distance for measuring convergence is the so-called $\chi$-square distance:

$$
\Delta^{\prime}(s)=\max _{y \in V(G)}\left(\sum_{x \in V(G)} \frac{\left(P^{s}(y, x)-\phi(x)\right)^{2}}{\phi(x)}\right)^{1 / 2}
$$


It is easy to see that $\Delta_{T V}(s) \leq \frac{1}{2} \Delta^{\prime}(s)$. Thus a convergence upper bound for $\Delta^{\prime}(s)$ implies one for $\Delta_{T V}(s)$.

Now we consider the characteristic vector $\chi_{x}$ defined by:

$$
\chi_{x}(y)= \begin{cases}1 & \text { if } y=x \\ 0 & \text { otherwise. }\end{cases}
$$

Let $P_{0}$ denote the projection to the eigenspace associated with 1. (Namely, $\left.P_{0}=\mathbf{1} \cdot \phi\right)$. We can then rewrite the $\chi$-square distance as follows:

$$
\begin{aligned}
\Delta^{\prime}(t)^{2} & =\max _{y}\left\|\left(\chi_{y} P^{t}-\phi\right) \Phi^{-1 / 2}\right\|^{2} \\
& =\max _{y}\left\|\left(\chi_{y}-\phi\right) P^{t} \Phi^{-1 / 2}\right\|^{2}
\end{aligned}
$$

where $\phi$ is the Perron vector and $\Phi$ is the diagonal matrix with $\Phi(v, v)=\phi(v)$.

The Perron vector $\phi$ is the stationary distribution if $G$ is strongly connected and aperiodic (i.e., the g.c.d. of all cycle lengths is 1). As it turns out (similar to the undirected case), periodicity is not an absolute obstacle for the mixing of random walks. We can modify our random walk $P$ by using a lazy walk, denoted by $\mathcal{P}$ for which with probability $1 / 2$ we choose to stay at the current vertex. In other words, the transition probability matrix of the lazy walk is taken to be

$$
\mathcal{P}=\frac{I+P}{2} .
$$

Suppose $P$ has left eigenvalues $\rho_{0}=1$ and $\rho_{1}, \ldots, \rho_{n-1}$, where $\rho_{i}$ are complex numbers (as roots of the characteristic polynomials). From the PerronFrobenius theorem, $\left|\rho_{i}\right| \leq 1$ for all $i$. The lazy walk $\mathcal{P}$ still has left eigenvector $\phi$ and has eigenvalues $\left(1+\rho_{i}\right) / 2$. Since $\left|\left(1+\rho_{i}\right) / 2\right|<1$ for any $i \neq 0, \mathcal{P}$ is not periodic. Therefore we have the following.

Theorem 5 In a directed graph $G$ with Laplacian eigenvalues $0=\lambda_{0} \leq \lambda_{1} \leq$ $\ldots \leq \lambda_{n-1}$, there is a lazy random walk with the transition probability matrix $\mathcal{P}$ having eigenvalues $\rho_{i}^{\prime}$ satisfying:

$$
\frac{\lambda_{1}}{4} \leq \min _{i \neq 0}\left(1-\left|\rho_{i}^{\prime}\right|\right) .
$$

Proof: $\quad$ Since $\mathcal{P}=(I+P) / 2$, we have $\rho_{i}^{\prime}=\left(1+\rho_{i}\right) / 2$, and so,

$$
\begin{aligned}
\left|\rho_{i}^{\prime}\right|^{2} & =\left|\frac{1+\rho_{i}}{2}\right|^{2} \\
& \leq \frac{\left(1+\operatorname{Re} \rho_{i}\right)^{2}}{4}+\frac{\left(\operatorname{Im} \rho_{i}\right)^{2}}{4} \\
& \leq \frac{1+\operatorname{Re} \rho_{i}}{2}
\end{aligned}
$$


implying

$$
\begin{aligned}
1-\left|\rho_{i}^{\prime}\right| & \geq 1-\sqrt{\frac{1+\operatorname{Re} \rho_{i}}{2}} \\
& =\frac{1-\frac{1+\operatorname{Re} \rho_{i}}{2}}{1+\sqrt{\frac{1+\operatorname{Re} \rho_{i}}{2}}} \\
& \geq \frac{1-\operatorname{Re} \rho_{i}}{4} .
\end{aligned}
$$

Thus From Theorem 2, we have

$$
\begin{aligned}
\min _{i \neq 0}\left(1-\left|\rho_{i}^{\prime}\right|\right) & \geq \min _{i \neq 0} \frac{1-\operatorname{Re} \rho_{i}}{4} \\
& \geq \frac{\lambda_{1}}{4} .
\end{aligned}
$$

We note that the statements in the above theorem does not hold if the word "lazy" is omitted. For example, for a random walk on the directed cycle $C_{n}$, the eigenvalue $\lambda_{1}$ of the Laplacian is strictly larger than $\min _{i \neq 0}\left(1-\left|\rho_{i}\right|\right)=0$.

Theorem 6 Suppose that a strongly connected directed graph $G$ on $n$ vertices has Laplacian eigenvalues $0=\lambda_{0} \leq \lambda_{1} \leq \ldots \leq \lambda_{n-1}$. Suppose that $G$ has transition probability matrix $P$ and a lazy random walk $\mathcal{P}=(I+P) / 2$. Then the matrix $M=\Phi^{1 / 2} \mathcal{P} \Phi^{-1 / 2}$ satisfies

$$
\frac{\|f M\|^{2}}{\|f\|^{2}} \leq 1-\frac{\lambda_{1}}{2}
$$

for all vectors $f$ satisfying $f \Phi^{1 / 2} \mathbf{1}=0$.

Proof: The vector $f_{0}$ with $f_{0}(v)=\sqrt{\phi(v)}$ is a left eigenvector of $M$ with eigenvalue 1 . We consider vectors $f$ orthogonal to $f_{0}$, i.e., $f \Phi^{1 / 2} \mathbf{1}=0$. We have

$$
\begin{aligned}
\frac{\|f M\|^{2}}{\|f\|^{2}} & =\frac{f M M^{*} f^{*}}{f f^{*}} \\
& =\frac{f \Phi^{1 / 2} \mathcal{P} \Phi^{-1} \mathcal{P}^{*} \Phi^{1 / 2} f^{*}}{f f^{*}} \\
& =\frac{f \Phi^{1 / 2}(I+P) \Phi^{-1}\left(I+P^{*}\right) \Phi^{1 / 2} f^{*}}{4 f f^{*}} .
\end{aligned}
$$

Now we define $h=f \Phi^{-1 / 2}$ and then $h$ is orthogonal to $\phi$. By substituting $h$ in 
the above equation, we have

$$
\begin{aligned}
\frac{\|f M\|^{2}}{\|f\|^{2}} & =\frac{h \Phi(I+P) \Phi^{-1}\left(I+P^{*}\right) \Phi h^{*}}{4 h \Phi h^{*}} \\
& =\frac{h\left(\Phi+\Phi P+P^{*} \Phi+\Phi P \Phi^{-1} P^{*} \Phi\right) h^{*}}{4 h \Phi h^{*}} \\
& =\frac{h\left[\left(-2 \Phi+\Phi P+P^{*} \Phi\right)+\left(3 \Phi+\Phi P \Phi^{-1} P^{*} \Phi\right)\right] h^{*}}{4 h \Phi h^{*}} \\
& =-\frac{R(h)}{4}+\left(3+\frac{\left\|f \Phi^{1 / 2} P \Phi^{-1 / 2}\right\|^{2}}{\|f\|^{2}}\right) \frac{1}{4} \\
& \leq-\frac{R(h)}{4}+1 \\
& \leq 1-\frac{\lambda_{1}}{2}
\end{aligned}
$$

as desired.

Theorem 7 Suppose that a strongly connected directed graph $G$ on $n$ vertices has Laplacian eigenvalues $0=\lambda_{0} \leq \lambda_{1} \leq \ldots \leq \lambda_{n-1}$. Then $G$ has a lazy random walk with the rate of convergence of order $2 \lambda_{1}^{-1}\left(-\log \min _{x} \phi(x)\right)$. Namely, after at most $t \geq 2 \lambda_{1}^{-1}\left(\left(-\log \min _{x} \phi(x)\right)+2 c\right)$ steps, we have

$$
\Delta^{\prime}(t) \leq e^{-c} .
$$

Proof: From (3), the rate of convergence of $\mathcal{P}=(I+P) / 2$ in $\chi$-square distance can be computed as follows:

$$
\begin{aligned}
\Delta^{\prime}(t) & =\max _{y}\left\|\left(\chi_{y}-\phi\right) \mathcal{P}^{t} \Phi^{-1 / 2}\right\| \\
& =\max _{y}\left\|\left(\chi_{y}-\phi\right) \Phi^{-1 / 2}\left(\Phi^{1 / 2} \mathcal{P}^{t} \Phi^{-1 / 2}\right)\right\| \\
& =\max _{y}\left\|g \Phi^{1 / 2} \mathcal{P}^{t} \Phi^{-1 / 2}\right\| \\
& =\max _{y}\left\|g M^{t}\right\|
\end{aligned}
$$

where $g=\left(\chi_{y}-\phi\right) \Phi^{-1 / 2}$ is orthogonal to $\phi^{1 / 2}$ and $M$ denotes $\Phi^{1 / 2} \mathcal{P} \Phi^{-1 / 2}$. Using Theorem 6 (which gives $\left\|f M^{k}\right\|^{2}=\left\|f M^{k-1} M\right\|^{2} \leq\left(1-\lambda_{1} / 2\right)\left\|f M^{k-1}\right\|^{2}$ ), we have

$$
\begin{aligned}
\Delta^{\prime}(t)^{2} & =\max _{y}\left\|\left(\chi_{y}-\phi\right) \Phi^{-1 / 2} M^{t}\right\|^{2} \\
& \leq\left(1-\lambda_{1} / 2\right)^{t} \max _{y}\left\|\left(\chi_{y}-\phi\right) \Phi^{-1 / 2}\right\|^{2} \\
& \leq\left(1-\lambda_{1} / 2\right)^{t} \max _{y} \phi(y)^{-1} .
\end{aligned}
$$


Therefore we have

$$
\begin{aligned}
\Delta^{\prime}(t) & \leq \frac{\left(1-\lambda_{1} / 2\right)^{t / 2}}{\sqrt{\min _{x} \phi(x)}} \\
& \leq e^{-t \lambda_{1} / 4-\frac{1}{2} \log \left(\min _{x} \phi(x)\right)} \\
& \leq e^{-c}
\end{aligned}
$$

provided $t \geq 2 \lambda_{1}^{-1}\left(\left(-\log \left(\min _{x} \phi(x)\right)\right)+2 c\right)$.

\section{Comparison theorems}

As in the undirected graphs, we can bound the Laplacian eigenvalues of one directed graph by the Laplacian eigenvalues of another provided each directed edge in the first graph is associated with "short" directed paths in the second graph. Many applications of such comparison techniques are given in Diaconis and Stroock [7] and numerous other papers $[8,10]$ in particular for card shuffling games. These comparison theorems are sometimes called "Poincaré" inequalities [7]. The proof here is quite similar to that for the undirected case as given in $[6]$.

Theorem 8 Let $G$ and $G^{\prime}$ be two strongly connected directed graphs, with Laplacian eigenvalues $\lambda_{1}$ and $\lambda_{1}^{\prime}$, respectively. Suppose that the vertex set of $G$ can be embedded onto the vertex set of $G^{\prime}$ under the mapping $\varphi: V(G) \rightarrow V\left(G^{\prime}\right)$, satisfying the following conditions for fixed positive values a, $l, m$ :

(a) Each edge $(x, y)$ in $E(G)$ is associated with a path, denoted by $Q_{x, y}$, joining $\varphi(x)$ to $\varphi(y)$ in $G^{\prime}$ of length at most $l$.

(b) Let $\phi, \phi^{\prime}$ denote the Perron vectors of $G$ and $G^{\prime}$, respectively. For any $v$ in $V\left(G^{\prime}\right)$, we have

$$
\sum_{x \in \varphi^{-1}(v)} \phi(x) \geq a \phi^{\prime}(v) .
$$

(c) There is a postive constant $m$ such that each edge $e=(u, v)$ in $G^{\prime}$ satisfies

$$
m \phi^{\prime}(u) P^{\prime}(u, v) \geq \sum_{\substack{x, y \\ e \in Q_{x, y}}} \phi(x) P(x, y) .
$$

Then we have

$$
\lambda_{1}^{\prime} \geq \frac{a \lambda_{1}}{l m} .
$$


Proof: Let $f^{\prime}$ denote the vector achieving the Rayleigh quotient as in Theorem 1 for $\lambda_{1}^{\prime}$ :

$$
\lambda_{1}^{\prime}=\frac{R\left(f^{\prime}\right)}{2}
$$

and $\sum_{v} f^{\prime}(v) \phi^{\prime}(v)=0$.

We define $f: V(G) \rightarrow \mathbb{R}$ as follows: For a vertex $x$ in $V(G)$,

$$
f(x)=f^{\prime}(\varphi(x))-c
$$

where the constant $c$ is chosen to satisfy

$$
\sum_{x} f(x) \phi(x)=0
$$

We note that

$$
\begin{aligned}
\sum_{x \in V(G)} f^{2}(x) \phi(x) & \left.=\sum_{x \in V(G)}\left(f^{\prime}(\varphi(x))-c\right)\right)^{2} \phi(x) \\
& =\sum_{v \in V\left(G^{\prime}\right)}\left(f^{\prime}(v)-c\right)^{2} \sum_{\varphi^{-1}(v)=x} \phi(x) \\
& \geq a \sum_{v \in V\left(G^{\prime}\right)}\left(f^{\prime}(v)-c\right)^{2} \phi^{\prime}(v) .
\end{aligned}
$$

Now, for $(x, y) \in E(G)$ with $\varphi(x)=u, \varphi(y)=v$, let $Q_{x, y}$ denote the path associated with $(x, y)$ joining $u$ and $v$ in $G^{\prime}$. We have

$$
\begin{aligned}
(f(x)-f(y))^{2} & =\left(f^{\prime}(u)-f^{\prime}(v)\right)^{2} \\
& \leq\left|Q_{x, y}\right| \sum_{e \in Q_{x, y}} f^{\prime}(e)^{2} \\
& \leq l \sum_{e \in Q_{x, y}} f^{\prime}(e)^{2},
\end{aligned}
$$

where $f^{\prime}(e)^{2}=\left(f^{\prime}(a)-f^{\prime}(b)\right)^{2}$ for $e=(a, b)$. Hence we have

$$
\begin{aligned}
m \sum_{e \in E\left(G^{\prime}\right)} f^{\prime}(e)^{2} \phi^{\prime}(u) P^{\prime}(u, v) & \geq \sum_{(x, y) \in E(G)} \sum_{e \in Q_{x, y}} f^{\prime}(e)^{2} \phi(x) P(x, y) \\
& \geq \frac{1}{l} \sum_{(x, y) \in E(G)}(f(x)-f(y))^{2} \phi(x) P(x, y) .(5)
\end{aligned}
$$


Combining inequalities (4),(5), we have

$$
\begin{aligned}
2 \lambda_{1}^{\prime}= & \sup _{c^{\prime}} \frac{\sum_{(u, v) \in E\left(G^{\prime}\right)}\left(f^{\prime}(u)-f^{\prime}(v)\right)^{2} \phi^{\prime}(u) P^{\prime}(u, v)}{\sum_{v \in V\left(G^{\prime}\right)}\left(f^{\prime}(v)-c^{\prime}\right)^{2} \phi^{\prime}(v)} \\
\geq & \frac{\sum_{(u, v) \in E\left(G^{\prime}\right)}\left(f^{\prime}(u)-f^{\prime}(v)\right)^{2} \phi^{\prime}(u) P^{\prime}(u, v)}{\sum_{v \in V\left(G^{\prime}\right)}\left(f^{\prime}(v)-c\right)^{2} \phi^{\prime}(v)} \\
= & \frac{\sum_{(u, v) \in E\left(G^{\prime}\right)}\left(f^{\prime}(u)-f^{\prime}(v)\right)^{2} \phi^{\prime}(u) P^{\prime}(u, v)}{\sum_{(x, y) \in E(G)}(f(x)-f(y))^{2} \phi(x) P(x, y)} \cdot \frac{\sum_{(x, y) \in E(G)}(f(x)-f(y))^{2} \phi(x) P(x, y)}{\sum_{x \in V(G)} f^{2}(x) \phi(x)} \\
& \frac{\sum_{v \in V(G)}\left(f^{\prime}(v)-c\right)^{2} \phi^{\prime}(v)}{\sum_{v\left(G^{\prime}\right)}(f(x)-f(y))^{2} \phi(x) P(x, y)} \\
\geq & \frac{1}{m l} \cdot \frac{(x, y) \in E(G)}{\sum_{x \in V(G)} f^{2}(x) \phi(x)}
\end{aligned}
$$

Since

$$
\sum_{x} f(x) \phi(x)=0
$$

we have

$$
\frac{\sum_{(x, y) \in E(G)}(f(x)-f(y))^{2} \phi(x) P(x, y)}{\sum_{x \in V(G)} f^{2}(x) \phi(x)} \geq 2 \lambda_{1}
$$

Hence

$$
\lambda_{1}^{\prime} \geq \frac{a}{m l} \lambda_{1}
$$

and the proof of Theorem 8 is complete.

Theorem 9 Let $G$ and $G^{\prime}$ be two connected regular graphs with eigenvalues $\lambda_{1}$ and $\lambda_{1}^{\prime}$ and degrees $k$ and $k^{\prime}$, respectively. Suppose that the vertex set of $G$ is the same as the vertex set of $G^{\prime}$. We assume that for each edge $\{x, y\}$ in $G$, there is a path $P(x, y)$ in $G^{\prime}$ joining $x$ and $y$ of length at most $l$. Furthermore, 
suppose that every edge in $G^{\prime}$ is contained in at most $t$ paths $P(x, y)$. Then we have

$$
\lambda_{1}^{\prime} \geq \frac{\lambda_{1}}{t l} .
$$

Proof: Since $G$ is regular, for an edge $(x, y)$, we have

$$
\phi(x)=\frac{1}{n}, \quad \text { and } \quad P(x, y)=\frac{1}{k} .
$$

Thus the condition (c) in Theorem 8 is satisfied if we choose

$$
m=\frac{t k^{\prime}}{k} .
$$

Condition (b) is satisfied with $a=1$. Condition (a) is one of the assumptions. This gives

$$
\lambda_{1}^{\prime} \geq \frac{\lambda_{1}}{t l}
$$

as desired.

\section{Polynomial versus exponential convergence rates for various families of directed graphs}

Unlike undirected graphs, some directed graphs can have random walks which require an exponential number of steps to converge to the stationary distribution (in total variation distance or in relative pointwise distance). Here we summarize convergence bounds for various families of directed graphs.

Theorem 10 A strongly connected regular directed graph $G$ on $n$ vertices with out-degree $k$ has has a lazy random walk converging to the uniform distribution with the rate of convergence no more than $k^{2} n^{2} \log n$.

Proof: The proof follows from Theorem 7 and Lemma 2. Note that $\phi(x)=1 / n$ and also the eigenvalue $\lambda_{1}$ of the Laplacian satisfies

$$
\lambda_{1} \geq \frac{h(G)^{2}}{2} \geq \frac{2}{k^{2} n^{2}} .
$$

The convergence rate is bounded above by

$$
2 \lambda_{1}^{-1}\left(-\log \min _{x} \phi(x)\right) \leq k^{2} n^{2} \log n .
$$


Theorem 11 A strongly connected Eulerian directed graph $G$ with $m$ edges has a lazy random walk with the rate of convergence no more than $m^{2} \log m$.

Proof: The proof follows from Theorem 7 and Lemma 3 together with the fact that

$$
\phi(x) \geq \frac{1}{2 m}
$$

The eigenvalue $\lambda_{1}$ of the Laplacian satisfies

$$
\lambda_{1} \geq \frac{h(G)^{2}}{2} \geq \frac{2}{m^{2}} .
$$

The convergence rate is bounded above by

$$
2 \lambda_{1}^{-1}\left(-\log \min _{x} \phi(x)\right) \leq m^{2} \log m
$$

We remark that many random walk problems such as those originating from card shuffling are random walks for regular or Eulerian graphs. The above theorems imply polynomial upper bounds for their rates of convergence. For general directed graphs, $\phi(x)$ can be as small as $c^{-n}$ even for bounded degree graphs. As a result, the Cheeger constant $h_{G}$ can be exponentially small, and consequently so is the eigenvalue $\lambda_{1}$.

\section{References}

[1] D. Aldous and J. Fill, Reversible Markov chains and random walks on graphs, draft of a book.

[2] N. Alon, Eigenvalues and expanders, Combinatorica 6 (1986) 86-96.

[3] C. Asci, Generating uniform random vectors, J. Theoret. Probab. 14 (2001), 333-356.

[4] F. Bassiri, Random walks on finite groups of multiplicity two, Ph. D. Thesis, Harvard University, 1997.

[5] A. Björner and L. Lovász, Chip-firing games on directed graphs, J. of Algebraic Combinatorics 1 (1992) 305-328

[6] F. Chung, Spectral Graph Theory, AMS Publications, 1997.

[7] P. Diaconis and D.W. Stroock, Geometric bounds for eigenvalues of Markov chains, Annals Applied Prob. 1 (1991), 36-61.

[8] P. Diaconis and L. Saloff-Coste, Comparison theorems for reversible Markov chains, Annals of Applied Prob. 3 (1993), 696-730. 
[9] R. A. Horn and C. R. Johnson, Matrix Analysis, Cambridge University Press, Cambridge, 1990.

[10] J. A. Fill, Eigenvalue bounds on convergence to stationarity for nonreversible Markov chains, with an application to the exclusion process, Ann. Appl. Probab. 1 (1991), 62-87.

[11] M. Hildebrand, Rates of convergence for a non-reversible Markov chain sampler, preprint. 